\title{
Fatores associados ao risco de pé diabético em pessoas com diabetes mellitus na Atenção Primária*
}

\author{
Factors associated with the risk of diabetic foot in patients with diabetes mellitus in \\ Primary Care \\ Factores asociados al riesgo de pie diabético en personas con diabetes mellitus en la \\ Atención Primaria
}

Como citar este artigo:

Lira JAC, Nogueira LT, Oliveira BMA, Soares DR, Santos AMR, Araújo TME. Factors associated with the risk of diabetic foot in patients with diabetes mellitus in Primary Care. Rev Esc Enferm USP. 2021;55:e03757. https://doi.org/10.1590/S1980-220X2020019503757

\section{Jefferson Abraão Caetano Lira ${ }^{1}$ \\ Lídya Tolstenko Nogueira ${ }^{1}$ \\ Bianca Maria Aguiar de Oliveira ${ }^{1}$ \\ Débora dos Reis Soares ${ }^{1}$ \\ Ana Maria Ribeiro dos Santos ${ }^{1}$ \\ Telma Maria Evangelista de Araújo ${ }^{1}$ \\ * Extraído da dissertação "Avaliação do rastreamento e da monitorização de pessoas com pé diabético na Atenção Primária à Saúde", \\ Programa de Pós-Graduação em Enfermagem, \\ Universidade Federal do Piauí, 2019. \\ ${ }^{1}$ Universidade Federal do Piauí, \\ Teresina, PI, Brasil.}

\section{ABSTRACT}

Objective: To analyze factors associated with diabetic foot risk in patients with diabetes mellitus assisted in Primary Care. Method: Observational, analytic, and transversal study took place in Teresina, Piauí, with diabetic patients who are assisted in Primary Care. Data collection took place through interviews, foot clinical exams, and medical record analysis. We used the Mann-Whitney, Pearson's Chi-square and multiple logistic regression statistics tests to analyze the data. The association power among categorical variables was measured by Odds Ratio. Results: 322 patients participated. Marital status with a partner presented a protection factor $(p=0.007)$. Risk factors for the development of the diabetic foot are: arterial hypertension $(p=0.045)$, obesity $(p=0.011)$, smoking $(p=0.027)$, not being submitted to follow ups $(p=0.046)$, inadequate control of capillary blood glucose $(\mathrm{p}<0.001)$, indisposition to the care of the foot $(\mathrm{p}=0.014)$, and foot self-exam less frequently $(\mathrm{p}=0.040)$. Conclusion: Sociodemographic, clinical, and self-care aspects interfere in diabetic foot development, highlighting the necessity of effective follow up tracking and educational interventions for patients with diabetes mellitus in Primary Care.

\section{DESCRIPTORS}

Diabetes Mellitus; Diabetic Foot; Mass Screening; Primary Health Care; Nursing. 


\section{INTRODUÇÃO}

O pé diabético é uma síndrome que acomete os membros inferiores de pessoas com diabetes mellitus (DM), decorrente do tratamento não efetivo, repercutindo em complicações vasculares, nervosas, ulcerações e deformidades ${ }^{(1)}$. Esse agravo evitável é responsável pelo elevado número de amputações e internações hospitalares, gerando crescimento dos custos para os serviços de saúde, além de comprometer a produtividade e a qualidade de vida desses pacientes ${ }^{(2)}$.

$\mathrm{Na}$ Espanha, 73\% das amputações em membros inferiores foram devido ao DM, com incidência de 11,2 para 100.000 habitantes, no período de 2001 a $2014^{(3)}$. No Ceará, entre 2010 e 2015, foram registradas 4.182 internações hospitalares para terapêutica das complicações do pé diabético, apresentando correlação positiva e forte entre o número de internações e as amputações relacionadas ao $\mathrm{DM}^{(4)}$. No Piauí, constatou-se que $52 \%$ dos pacientes com DM apresentaram alteração na sensibilidade protetora plantar, um dos fatores preditivos para o pé diabético ${ }^{(5)}$.

O pé diabético ocorre devido ao descontrole metabólico, ao déficit de conhecimento e a não adesão à terapêutica recomendada. Além do mais, a higiene precária, a utilização de calçados inapropriados, o corte inadequado das unhas, a presença e o não tratamento de onicomicoses e onicocriptoses, a remoção incorreta de calosidades, o tratamento inadequado de úlceras neuroisquêmicas e a isquemia periférica são fatores agravantes para o pé diabético ${ }^{(6)}$.

Dessa forma, enfatiza-se a necessidade do rastreamento, que consiste no levantamento dos fatores de risco para o desenvolvimento do pé diabético, mediante exames clínicos e laboratoriais ${ }^{(1)}$. Por meio dessa estratégia, o enfermeiro implementa o plano de cuidados, as recomendações e os encaminhamentos que são imprescindíveis no gerenciamento do cuidado aos pacientes com $\mathrm{DM}^{(7)}$. No entanto, um estudo realizado na Paraíba constatou que a maior parte dos enfermeiros não realiza o exame clínico dos pés para avaliação da sensibilidade protetora plantar ${ }^{(8)}$.

Assim, o enfermeiro possui atuação significativa na assistência às pessoas com DM, pois apresenta competências e habilidades direcionadas ao cuidado e à promoção da saúde. Desse modo, a efetividade do rastreamento do pé diabético, por parte desse profissional, é crucial para melhorar o gerenciamento do cuidado e reduzir a ocorrência de agravo no âmbito da Atenção Primária ${ }^{(9)}$.

No intuito de investigar a operacionalização do rastreamento do pé diabético e contribuir para a instituição de uma linha de cuidados voltada para a integralidade da assistência às pessoas com DM, este estudo objetivou analisar os fatores associados ao risco de pé diabético em pessoas com diabetes mellitus atendidas na Atenção Primária.

\section{MÉTODO}

\section{Desenho do estudo}

Estudo observacional, analítico e transversal.

\section{Cenário}

O estudo foi realizado em Teresina, Piauí, na Atenção Primária, em seis Unidades Básicas de Saúde (UBS) da regional Centro-Norte, por apresentarem maior demanda de pessoas com DM no município.

\section{População e Amostra}

A população foi constituída de 2.015 pacientes com DM, que estavam cadastrados no Programa Hiperdia da Estratégia Saúde da Família da referida regional e que realizavam consultas de rotina nas UBS, em junho de 2018. A amostra foi composta de pacientes que atenderam aos critérios de inclusão: maiores de 18 anos e com diagnóstico de DM tipo 1 ou 2; e exclusão: aqueles com neuropatia atribuída a outros fatores. A fórmula de estimativa da proporção populacional para populações finitas foi utilizada para o cálculo da amostra mínima necessária, com nível de confiança de $95 \%$, prevalência presumida de $50 \%$ e erro máximo de 5\%, resultando em 322 pacientes. A seleção da amostra foi não probabilística por conveniência.

\section{Coleta de Dados}

Antes de ser iniciada a coleta, realizou-se pré-teste com 32 participantes para testar a aplicação dos instrumentos. A coleta de dados ocorreu no período de fevereiro a agosto de 2019, em três etapas.

A primeira etapa consistiu em entrevista utilizando formulário adaptado para levantar os aspectos sociodemográficos e clínicos ${ }^{(10)}$ e o Questionário de Adesão ao Autocuidado com os Pés para Diabéticos para avaliar as práticas de autocuidado ${ }^{(11)}$. Ressalta-se que todas as variáveis referentes ao autocuidado dos pés, a exemplo da não disposição para cuidar dos pés, foram coletadas por meio de relato verbal do paciente em entrevista.

$\mathrm{Na}$ segunda etapa foi realizado o exame clínico dos pés por meio do Instrumento de Classificação de Neuropatia de Michigan (Michigan Neuropathy Screening Instrument MNSI), considerando neuropatia presente em escore igual ou superior a 2,5 , com pelo menos um sinal ou sintoma ${ }^{(12)}$. A classificação do risco de desenvolver o pé diabético foi realizada a partir do instrumento para exame do pé diabético em 3 minutos ${ }^{(13)}$. Foram consideradas pessoas com pé diabético aquelas que apresentaram ferida aberta ou área ulcerativa, com ou sem sinais de infecção nos pés.

Os pacientes foram devidamente orientados e posicionados, sentados na maca ou em cadeira, de maneira a facilitar a realização do exame. A demonstração prévia do procedimento era realizada para que os pacientes compreendessem os estímulos que poderiam sentir durante o exame dos pés, além de serem orientados a permanecerem com os olhos fechados no momento do exame, para prevenir resultados errados. Para realização dos testes, foram utilizados o monofilamento de SemmesWeinstein de $10 \mathrm{~g}$, o diapasão de $128 \mathrm{~Hz}$ e o martelo neurológico.

$\mathrm{Na}$ terceira etapa realizou-se a análise do prontuário para o levantamento de dados antropométricos e de exames laboratoriais. Enfatiza-se que os dados foram coletados nos dias de consulta do Programa Hiperdia, antes do atendimento dos pacientes, em sala reservada das UBS.

As variáveis independentes foram as sociodemográficas, as clínicas e as relacionadas ao autocuidado, relacionados ao risco de desenvolver o pé diabético. Cujo desfecho foi ter ou não risco de desenvolver o pé diabético. 


\section{ANÁLISE E TRATAMENTO DOS DADOS}

A tabulação dos dados, com digitação dupla, foi realizada no Microsoft Excel 2013. Posteriormente, os dados foram exportados para o Software Statistical Package for Social Science (SPSS), versão 22.0. Para a análise foi realizada estatísticas, tanto descritiva quanto inferencial. Na estatística descritiva, para as variáveis numéricas, foram calculados mediana, valor mínimo e valor máximo. Para as categóricas foram utilizados frequência absoluta e percentual. $\mathrm{Na}$ estatística inferencial foram empregados testes estatísticos bivariados e multivariados.

Para verificar o padrão de distribuição das variáveis numéricas, utilizou-se o teste de normalidade Kolmogorov-Smirnov, em que se evidenciou distribuição não normal. $\mathrm{Na}$ análise inferencial, para a estatística bivariada, empregaram-se os testes Mann-Whitney, para comparar uma variável numérica com uma categórica dicotômica, além do Qui quadrado de Pearson, para verificar a associação entre duas variáveis categóricas. A Odds Ratio (OR), com Intervalos de Confiança (IC) de 95\%, foi utilizada para verificar a força de associação entre as variáveis categóricas. Enfatiza-se que a categoria de referência adotada ocorreu com base na importância clínica.

$\mathrm{Na}$ estatística multivariada foi construído modelo de regressão logística múltipla pelo método stepwise forward, sendo introduzida, primeiramente, a variável de maior significância estatística e, posteriormente, as demais. Desse modo, seguindo a ordem decrescente de associação identificada na análise bivariada. $\mathrm{O}$ critério de inclusão das variáveis no modelo multivariado foi o valor de $\mathrm{p} \leq 0,20$ obtido na análise bivariada ${ }^{(14)}$. A multicolinearidade entre as variáveis independentes foi identificada pelo Variance Inflation Factor $(V I F)$, em que o valor superior a quatro foi adotado como ponto de corte para o diagnóstico de multicolinearidade $\mathrm{VIF}^{(15)}$. Consideraram-se significativos para todos os testes estatísticos utilizados os valores de $\mathrm{p}<0,05$.

\section{Aspectos éticos}

Este estudo respeitou os aspectos éticos da Resolução $n^{\circ}$ 466/2012 do Conselho Nacional de Saúde e foi aprovado pelo Comitê de Ética em Pesquisa da Universidade Federal do Piauí, em 2018, com o parecer n 2.817.426.

\section{RESULTADOS}

$\mathrm{Na}$ caracterização sociodemográfica, prevaleceram os idosos jovens $(53,4 \%)$, o sexo feminino $(70,2 \%)$, a cor parda (70,2\%), a situação conjugal com companheiro $(62,4 \%)$ e a renda pessoal de um salário mínimo $(64,0 \%)$. Grande parte era aposentada $(33,2 \%)$ e possuía ensino fundamental incompleto (44,4\%).

A respeito dos aspectos clínicos, predominou o DM tipo 2 (94,4\%), os medicamentos orais como tratamento predominante $(86,0 \%)$ e o controle glicêmico inadequado $(51,9 \%)$. Ademais, 58,7\% relataram que possuíam a doença há menos de 10 anos, 66,1\% tinham dislipidemia e 72,0\% hipertensão arterial. Entretanto, a maioria não apresentou retinopatia $(55,6 \%)$, nefropatia $(82,0 \%)$ e obesidade $(72,7 \%)$, além de não ser tabagista $(89,8 \%)$ e nem fazer o uso de álcool $(82,6 \%)$. Em contrapartida, $76,7 \%$ possuíam baixa acuidade visual.

Com relação ao rastreamento do pé diabético, 86,3\% das pessoas com DM nunca foram submetidas ao exame clínico dos pés, 59,0\% tinham neuropatia diabética, 3,1\% pé diabético e $69,6 \%$ risco de desenvolver o pé diabético. Além disso, 57,8\% relataram que não receberam qualquer tipo de monitoramento.

No exame clínico dos pés, o pé direito foi o que mais apresentou aparência anormal $(68,9 \%)$, sendo a pele seca e/ou calosidades a complicação mais comum $(57,1 \%)$ e 1,9\% apresentavam úlcera. A presença do reflexo do tornozelo $(57,1 \%)$ e a preservação da sensibilidade protetora plantar ao monofilamento de $10 \mathrm{~g}(68,3 \%)$ prevaleceram no pé direito. No entanto, o pé esquerdo foi o que mais teve casos de ausência da percepção de vibração do hálux ao diapasão de $128 \mathrm{~Hz}(42,9 \%)$.

A idade $(\mathrm{p}=0,046)$, o tempo de diagnóstico do DM $(\mathrm{p}<0,001)$, os anos de estudo $(\mathrm{p}=0,008)$ e a quantidade de complicações nos pés $(p<0,001)$ apresentaram significância estatística com o risco de desenvolver o pé diabético (Tabela 1).

Tabela 1 - Diferenças de variáveis sociodemográficas e clínicas em relação ao risco de desenvolver o pé diabético - Teresina, Piauí, Brasil, 2019.

\begin{tabular}{lcccc}
\hline Variáveis & Mediana & Mínimo & Máximo & $\boldsymbol{p}$ valor \\
\hline Idade em anos & 62,0 & 26,0 & 87,0 & $\mathbf{0 , 0 4 6}$ \\
Anos de estudo & 7,5 & 0,0 & 18,0 & $\mathbf{0 , 0 0 8}$ \\
Renda em reais & 998,0 & 0,0 & $5.998,0$ & 0,429 \\
Tempo de diagnóstico em anos & 6,5 & 0,0 & 63,0 & $<0,001$ \\
IMC & 27,9 & 19,1 & 46,4 & 0,195 \\
Quantidade de complicações nos pés & 2,0 & 0,0 & 8,0 & $<\mathbf{0 , 0 0 1}$ \\
\hline
\end{tabular}

Teste Mann-Whitney.

$\mathrm{n}=322$

Identificou-se que apresentar hipertensão arterial (OR:1,96; IC:1,17-3,26) e obesidade (OR:1,85; IC:1,04-3,29) aumentam as chances de desenvolver o pé diabético. Entretanto, a situação conjugal com companheiro (OR:0,70; IC:0,510,98) e a submissão ao rastreamento (OR:0,57; IC:0,33-0,99) se mostraram fatores de proteção, conforme a Tabela 2. 
Tabela 2 - Associação dos aspectos sociodemográficos e clínicos com o risco de desenvolver o pé diabético - Teresina, Piauí, Brasil, 2019.

\begin{tabular}{|c|c|c|c|c|c|}
\hline \multirow{3}{*}{ Variáveis } & \multicolumn{2}{|c|}{ Risco de pé diabético } & \multirow{3}{*}{ OR bruta } & \multirow{3}{*}{ IC $95 \%$} & \multirow{3}{*}{$p$ valor } \\
\hline & Sim & Não & & & \\
\hline & n (\%) & n (\%) & & & \\
\hline \multicolumn{6}{|l|}{ Situação conjugal } \\
\hline Com companheiro* & $148(73,6)$ & $53(26,4)$ & 0,70 & $0,51-0,98$ & 0,046 \\
\hline Sem companheiro & $76(62,8)$ & $45(37,2)$ & & & \\
\hline \multicolumn{6}{|l|}{ Tipo de diabetes } \\
\hline Diabetes tipo $1^{*}$ & $14(77,8)$ & $4(22,2)$ & 1,57 & $0,50-4,89$ & 0,436 \\
\hline Diabetes tipo 2 & $210(69,1)$ & $94(30,9)$ & & & \\
\hline \multicolumn{6}{|l|}{ Hipertensão arterial } \\
\hline Sim* & $171(73,7)$ & $61(26,3)$ & 1,96 & $1,17-3,26$ & 0,010 \\
\hline Não & $53(58,9)$ & $37(41,1)$ & & & \\
\hline \multicolumn{6}{|l|}{ Obesidade } \\
\hline Sim* & $69(78,4)$ & $19(21,6)$ & 1,85 & $1,04-3,29$ & 0,034 \\
\hline Não & $155(66,2)$ & $79(33,8)$ & & & \\
\hline \multicolumn{6}{|l|}{ Tabagismo } \\
\hline $\operatorname{Sim}^{*}$ & $28(84,8)$ & $5(15,2)$ & 2,66 & $0,99-7,10$ & 0,044 \\
\hline Não & $196(67,8)$ & $93(32,2)$ & & & \\
\hline \multicolumn{6}{|l|}{ Uso de álcool } \\
\hline Sim* & $38(67,9)$ & $18(32,1)$ & 0,91 & $0,49-1,69$ & 0,760 \\
\hline Não & $186(69,9)$ & $80(30,1)$ & & & \\
\hline \multicolumn{6}{|l|}{ Rastreamento } \\
\hline Sim* & $25(56,8)$ & $19(43,2)$ & 0,57 & $0,33-0,99$ & 0,048 \\
\hline Não & $199(71,6)$ & $79(28,4)$ & & & \\
\hline \multicolumn{6}{|l|}{ Monitorização } \\
\hline $\operatorname{Sim}^{*}$ & $92(67,6)$ & $44(32,4)$ & 0,86 & $0,53-1,38$ & 0,522 \\
\hline Não & $132(71,0)$ & $54(29,0)$ & & & \\
\hline
\end{tabular}

* Categoria de referência.

Teste Qui quadrado de Pearson.

$\mathrm{n}=322$

$\mathrm{Na}$ associação do autocuidado com o risco de desenvolver o pé diabético, o controle glicêmico inadequado (OR:3,47; IC:2,11-5,70), não sentir disposição para cuidar dos pés (OR:3,45; IC:1,73-6,87), o corte das unhas de forma não quadrada (OR:1,88; IC:1,16-3,07) e a não utilização de hidratante (OR:2,16; IC:1,33-3,50) aumentam as chances de desenvolver o pé diabético. $O$ autoexame dos pés $(\mathrm{p}<0,001)$, a checagem dos sapatos antes de calçar $(p=0,009)$, a secagem dos espaços entre os dedos $(\mathrm{p}=0,016)$ e a utilização de soluções caseiras para realização do curativo $(p=0,004)$ apresentaram associação estatisticamente significativa com o risco de desenvolver o pé diabético, de acordo com a Tabela 3.

Tabela 3 - Associação do autocuidado com o risco de desenvolver o pé diabético. Teresina, Piauí, Brasil, 2019.

\begin{tabular}{|c|c|c|c|c|c|}
\hline \multirow{3}{*}{ Variáveis } & \multicolumn{2}{|c|}{ Risco de pé diabético } & \multirow{3}{*}{ OR bruta } & \multirow{3}{*}{ IC $95 \%$} & \multirow{3}{*}{$p$ valor } \\
\hline & Sim & Não & & & \\
\hline & n (\%) & n (\%) & & & \\
\hline \multicolumn{6}{|c|}{$\begin{array}{l}\text { Procura o profissional de saúde ao } \\
\text { apresentar algum problema nos pés }\end{array}$} \\
\hline Não* & $123(72,8)$ & $46(27,2)$ & 1,38 & $0,86-2,22$ & 0,187 \\
\hline Sim & $101(66,0)$ & $52(34)$ & & & \\
\hline
\end{tabular}

continua... 
...continuação

\begin{tabular}{l} 
Variáveis \\
\hline $\begin{array}{l}\text { Alguém incentiva ou ajuda a realizar o } \\
\text { cuidado com os pés }\end{array}$
\end{tabular}

cuidado com os pés

$$
\begin{aligned}
& \text { Não* } \\
& \text { Sim }
\end{aligned}
$$

Pratica atividade física por, pelo menos, 30 minutos/dia

Nenhuma vez*

Pelo menos uma vez por semana

Controle dos níveis de glicemia capilar

Não*
Sim

Toma as medicações prescritas como recomendadas

Não*
Sim

O profissional de saúde orienta acerca dos cuidados com os pés

$$
\begin{aligned}
& \text { Não* } \\
& \text { Sim }
\end{aligned}
$$

Possui disposição para cuidar dos pés

Não*

Sim

Examina os pés frequentemente

Não

Semanalmente

Diariamente

Seca os espaços entre os dedos dos pés

Não

Semanalmente

Diariamente ou a cada banho

Faz a checagem dos sapatos antes de calçar

$$
\begin{aligned}
& \text { Não } \\
& \text { Raramente } \\
& \text { Sempre }
\end{aligned}
$$

Corta as unhas dos pés no formato quadrado

Nunca*

São cortadas quadradas, mas não se pode afirmar se sempre faz isso

Aplica hidratante nos pés

Nunca*

Quase sempre ou sempre

Faz curativo em casa com soluções caseiras ou outros produtos
Sim*
$63(82,9)$
$161(65,4)$

$13(17,1)$

2,56

$1,33-4,91$

0,004

Risco de pé diabético

\begin{tabular}{cc}
\hline Sim & Não \\
\hline $\mathbf{n}(\%)$ & n (\%)
\end{tabular}

$130(69,9) \quad 56(30,1)$

$50(27,3)$

1,4

$0,87-2,26$

0,164

$91(65,5)$

$48(34,5)$

$154(80,2)$

$38(19,8)$

3,47

$2,11-5,70$

$<0,001$

$70(53,8)$

$60(46,2)$

$5(23,8)$

1,43

$0,51-4,02$

0,495

$208(69,1)$

$93(30,9)$

$65(29,0)$

1,24

$0,75-2,07$

$65(66,3)$

$33(33,7)$

$68(86,1)$

$11(13,9)$

3,45

$1,73-6,87$

$<0,001$

$87(35,8)$

$30(19,7)$

$40(36,0)$

$28(47,5)$

$100(77,5)$

$29(22,5)$

$34(31,8)$

$34(31,8)$

$65(79,3)$

$17(20,7)$

$26(26,3)$

$73(73,7)$

$55(39,0)$

$85(34,6)$

$<0,001$

0,016

0,009

* Categoria de referência.

Teste Qui quadrado de Pearson.

$\mathrm{n}=322$ 
No modelo final de regressão logística múltipla, a situação conjugal com companheiro foi um fator de proteção para o desenvolvimento do pé diabético (ORa:0,47; IC:0,27-0,81). O tempo de diagnóstico do DM (ORa:0,92; IC:0,88-0,95) e a quantidade de complicações nos pés (ORa:0,63; IC:0,51-0,77) tiveram associação negativa, ou seja, as pessoas com menor tempo de diagnóstico e menor número de complicações possuem menores chances de desenvolverem o pé diabético. Apresentar hipertensão arterial (ORa:1,83; IC:1,01-3,32), obesidade (ORa:2,27; IC:1,21-4,28), ser tabagista (ORa:3,18; IC:1,14-8,86), não ter sido submetido ao rastreamento (ORa:2,1; IC:1,01-4,39), o controle inadequado dos níveis de glicemia capilar (ORa:3,02; IC:1,74-5,25), não sentir disposição para cuidar dos pés (ORa:2,90; IC:1,24-6,79) e a não realização do autoexame dos pés com frequência (ORa:2,11; IC:1,03-4,32) aumentam as chances de desenvolver o pé diabético (Tabela 4).

Tabela 4 - Regressão logística múltipla dos aspectos sociodemográficos, clínicos e de autocuidado com o risco de desenvolver o pé diabético. Teresina, Piauí, Brasil, 2019.

\begin{tabular}{|c|c|c|c|}
\hline Variáveis & OR ajustada & IC $95 \%$ & $p$ valor* \\
\hline Idade em anos & 0,99 & $0,96-1,01$ & 0,604 \\
\hline Anos de estudo & 1,04 & $0,97-1,11$ & 0,201 \\
\hline \multicolumn{4}{|l|}{ Situação conjugal } \\
\hline Com companheiro & 0,47 & $0,27-0,81$ & 0,007 \\
\hline Tempo de diagnóstico em anos & 0,92 & $0,88-0,95$ & $<0,001$ \\
\hline \multicolumn{4}{|l|}{ Hipertensão arterial } \\
\hline Sim & 1,83 & $1,01-3,32$ & 0,045 \\
\hline \multicolumn{4}{|l|}{ Obesidade } \\
\hline Sim & 2,27 & $1,21-4,28$ & 0,011 \\
\hline \multicolumn{4}{|l|}{ Tabagismo } \\
\hline Sim & 3,18 & $1,14-8,86$ & 0,027 \\
\hline \multicolumn{4}{|l|}{ Rastreamento } \\
\hline Não & 2,10 & $1,01-4,39$ & 0,046 \\
\hline \multicolumn{4}{|l|}{ Controle dos níveis de glicemia capilar } \\
\hline Não & 3,02 & $1,74-5,25$ & $<0,001$ \\
\hline \multicolumn{4}{|l|}{ Possui disposição para cuidar dos pés } \\
\hline Não & 2,90 & $1,24-6,79$ & 0,014 \\
\hline \multicolumn{4}{|l|}{ Examina os pés frequentemente } \\
\hline Não & 2,11 & $1,03-4,32$ & 0,040 \\
\hline \multicolumn{4}{|l|}{ Seca os espaços entre os dedos dos pés } \\
\hline Não & 1,03 & $0,48-2,19$ & 0,928 \\
\hline \multicolumn{4}{|c|}{ Faz a checagem dos sapatos antes de calçar } \\
\hline Não & 0,98 & $0,42-2,30$ & 0,977 \\
\hline \multicolumn{4}{|c|}{ Corta as unhas dos pés no formato quadrado } \\
\hline Nunca & 0,55 & $0,27-1,12$ & 0,103 \\
\hline \multicolumn{4}{|l|}{ Aplica hidratante nos pés } \\
\hline Nunca & 1,00 & $0,51-1,96$ & 0,989 \\
\hline \multicolumn{4}{|c|}{ Faz curativo em casa com soluções caseiras ou outros produtos } \\
\hline $\operatorname{Sim}$ & 1,86 & $0,85-4,03$ & 0,117 \\
\hline Quantidade de complicações nos pés & 0,63 & $0,51-0,77$ & $<0,001$ \\
\hline
\end{tabular}

*Regressão logística múltipla

$\mathrm{n}=322$

\section{DISCUSSÃO}

Nesta pesquisa, a caracterização sociodemográfica converge, em parte, com o estudo realizado no Paraná, no qual a maior parte dos pacientes com DM apresentavam média de 60 anos de idade, sexo feminino (62,0\%), 69,6\% possuíam companheiro e $67,7 \%$ apresentavam baixo nível de escolaridade, com menos de seis anos de estudo. Todavia, divergiu quanto a cor da pele, a qual prevaleceu a branca $(71,8 \%)^{(16)}$. 
A situação conjugal com companheiro, no modelo multivariado, mostrou-se fator de proteção para o desenvolvimento do pé diabético, o que pode ser explicado em decorrência do cônjuge oferecer suporte emocional e social no enfrentamento do $\mathrm{DM}$, melhorando a adesão do parceiro à terapêutica, mediante monitoramento do controle glicêmico, tomada das medicações prescritas, instituição das ações de autocuidado e de mudanças no estilo de vida.

$\mathrm{Na}$ Irlanda, a idade avançada, o sexo masculino, o histórico de tabagismo, o menor nível de atividade física e o diagnóstico de colesterol alto foram preditores de complicações macrovasculares. Outrossim, o diagnóstico de DM com 10 anos ou mais, o histórico de tabagismo e o diagnóstico de hipertensão arterial foram associados com o risco aumentado de complicações microvasculares ${ }^{(17)}$. Esses resultados corroboraram, parcialmente, com a presente pesquisa, pois a hipertensão arterial e o tabagismo aumentaram as chances para o desenvolvimento do pé diabético, enquanto a idade e a escolaridade não apresentaram associação estatisticamente significativa.

Estudo transversal realizado na Arábia Saudita também constatou que o DM tipo 2 foi o mais prevalente $(89,1 \%)$ e que os hipoglicemiantes orais eram a forma de tratamento medicamentoso em $57,2 \%$ dos pacientes. Em relação às comorbidades associadas, o percentual de nefropatia foi similar a esta pesquisa (7,6\%). No entanto, a hipertensão arterial $(47,2 \%) \mathrm{e}$ a dislipidemia (16,7\%) se mostraram divergentes, com valores inferiores aos registrados neste estudo ${ }^{(18)}$. Enfatiza-se que o $\mathrm{DM}$ associado à hipertensão arterial e à dislipidemia repercutem no aumento do risco cardiovascular e, por conseguinte, no aumento do risco de desenvolver o pé diabético.

O exame clínico dos pés, a partir da anamnese e do exame físico, é crucial para prevenção de ulceração e de amputação em membros inferiores em pessoas com DM, entretanto, a expressiva maioria dos pacientes nesta pesquisa relatou que nunca foi submetido ao exame clínico dos pés. Esse dado denuncia a falta de integralidade no atendimento às pessoas com DM, realçando a necessidade da implantação de uma linha de cuidados mais robusta, para sanar essa problemática na Atenção Primária.

Observou-se que a maioria teve escore positivo para neuropatia diabética. Mesmo que a maior parte não tenha apresentado pé diabético, o percentual de risco de desenvolver o pé diabético foi significativo, o que reforça a necessidade de realização do exame clínico dos pés, da investigação e do controle dos fatores de risco na Atenção Primária. Em convergência com esses resultados, uma pesquisa realizada Distrito Federal identificou que $41,9 \%$ tinham polineuropatia diabética dolorosa e $86,6 \%$ contavam com risco de ulceração nos pés ${ }^{(7)}$.

A pele seca, ocasionada por falta de hidratação, e as calosidades, devido ao atrito e à pressão plantar em decorrência de calçados inadequados, foram as principais complicações identificadas. $\mathrm{O}$ uso diário de creme hidratante previne a pele seca e as fissuras ${ }^{(10)}$. Já os calçados sem costuras, confortáveis e de numeração compatível são os mais adequados, porque os de bico fino e chinelos de dedo ocasionam pontos de pressão nos pés, o que pode repercutir em calosidades e ferimentos ${ }^{(19)}$. Essas orientações de autocuidado devem ser intensificadas pelo enfermeiro durante o atendimento aos pacientes com DM.

O exame para verificar a sensibilidade vibratória, por meio do diapasão de $128 \mathrm{~Hz}$, foi o que mais identificou alterações em ambos os pés. Apesar de ser eficaz no rastreamento do risco de desenvolver o pé diabético e recomendado pelo Ministério da Saúde ${ }^{(1)}$, observou-se que esse dispositivo não é disponibilizado aos enfermeiros da Atenção Primária, no cenário da pesquisa, o que dificulta a realização completa do exame clínico dos pés nos pacientes com DM.

O tempo de diagnóstico do DM, no modelo multivariado, apresentou associação estatística significativa com o risco de desenvolver o pé diabético, pois aqueles com menor tempo de doença tiveram menor risco de desenvolver o pé diabético. Sabe-se que o quadro de hiperglicemia, a longo prazo, compromete os sistemas nervoso e cardiovascular, por isso os pacientes com maior tempo de diagnóstico da doença estão mais propensos a complicações nos pés ${ }^{(20-21)}$.

As pessoas obesas apresentaram 2,1 vezes mais chances de desenvolver o pé diabético. De fato, o excesso de peso é um dos principais fatores que contribui para a manutenção da hiperglicemia, a partir de inúmeros mecanismos fisiológicos, a exemplo do aumento dos ácidos graxos livres na corrente sanguínea, da diminuição da adiponectina e da secreção de citocinas pelo tecido adiposo, o que ocasiona a resistência celular à insulina ${ }^{(22-24)}$.

Houve associação do número de complicações nos pés com o risco de desenvolver o pé diabético. Corroborando com esse resultado, um estudo identificou que os pacientes diabéticos com complicações nos pés tiveram 29,85\% mais chances de ulceração, se comparados aqueles sem anormalidades. Em relação às complicações, as calosidades plantares, também evidenciadas na presente pesquisa, foram um dos principais fatores de risco para ulceração nos pés ${ }^{(25)}$.

A não realização do rastreamento mostrou-se fator de risco para o desenvolvimento do pé diabético. E isso é explicado devido à maioria dos pacientes com $\mathrm{DM}$, no cenário teresinense, não ser submetida ao exame clínico dos pés de rotina. Além disso, constatou-se que a maioria não participou de monitorização, mediante estratificação do risco e encaminhamento ao especialista, o que dificulta a continuidade da assistência. Essa precariedade na assistência, ainda sem evolução, foi retratada em 2011 . Ocasião em que 79,5\% dos pacientes com DM afirmaram que não foram submetidos ao exame clínico dos pés durante o atendimento e $96,4 \%$ não realizaram o teste de sensibilidade dos pés nos últimos 12 meses $^{(26)}$.

O controle glicêmico apresentou associação com o risco de desenvolver o pé diabético, pois os pacientes com controle glicêmico inadequado tiveram 3,02 mais chances de desenvolverem o pé diabético. Estudo realizado em Pernambuco constatou que o controle glicêmico inadequado foi mais frequente entre pessoas com DM que declararam possuir três ou mais complicações da doença, assim como naqueles que, nos últimos 12 meses, não foram encaminhados ao endocrinologista ou cardiologista. $O$ descontrole glicêmico também foi mais prevalente quando o tratamento medicamentoso se 
tornava mais complexo, enfatizando que a orientação medicamentosa, por parte do enfermeiro, é imprescindível ${ }^{(27)}$.

A não inspeção diária dos pés pelos pacientes com $\mathrm{DM}$ teve associação estatisticamente significativa com risco de desenvolver o pé diabético. Pesquisa realizada na Espanha verificou que apenas $48,0 \%$ dos participantes conheciam o autocuidado específico para a prevenção do pé diabético, $50,6 \%$ realizavam a inspeção diária, 97,0\% lavavam os pés diariamente, 45,2\% hidratavam os pés e $65,7 \%$ cortavam as unhas adequadamente, salientando que o déficit de autocuidado com os pés também é um problema vivenciado nos países desenvolvidos ${ }^{(28)}$.

Em Taiwan, 62,8\% dos pacientes com DM nunca monitoraram o nível de glicose no sangue, quando tiveram úlceras nos pés, e $63,8 \%$ nunca procurariam tratamento para ulceração, se essa não fosse dolorosa. Além do mais, o comportamento de autocuidado com os pés, o não tratamento adequado e o status financeiro ruim influenciaram o autogerenciamento do DM por parte dos pacientes ${ }^{(29)}$. Embora não procurar os profissionais de saúde em caso de ulceração, no modelo bivariado, e realizar curativos em casa com soluções caseiras, no modelo multivariado, não tenham apresentado significância estatística para o desenvolvimento do pé diabético. Vale ressaltar que uma parcela considerável de pacientes, neste estudo, faziam essas ações errôneas.

Não sentir disposição para cuidar dos pés apresentou associação estatisticamente significativa e com o risco de desenvolver o pé diabético. Isso pode ser explicado devido à disposição ser uma variável que impulsiona as demais ações de autocuidado com os pés. Apesar da secagem dos espaços entre os dedos, da checagem dos sapatos antes de calçar, do corte no formato quadrado das unhas e da utilização de hidratante não terem associação com o risco de desenvolver o pé diabético, no modelo multivariado, esses cuidados são fundamentais para manter os pés saudáveis.

Ao comparar o diagnóstico situacional referente ao autocuidado com pés em pessoas diabéticas da capital piauiense com o interior do estado, observou-se, em estudo desenvolvido em Picos, Piauí, que 49,4\% dos pacientes com DM não sabiam realizar a higiene correta e nem o que deveriam observar no autoexame dos pés. Além do mais, $56,5 \%$ não conheciam o corte correto das unhas e desconheciam que os cuidados, a exemplo da lavagem, secagem, hidratação e massagem deveriam ser feitos juntos, mesmo $80 \%$ tendo disposição para realizar o autocuidado com os pés. Sendo assim, fica enfatizada a necessidade de estratégias educativas para sensibilizar os pacientes e incentivar os profissionais de saúde, a fim de garantir a eficácia na prevenção do pé diabético ${ }^{(30)}$.

Este estudo trouxe evidências para a enfermagem na Atenção Primária, pois constatou que a assistência às pessoas com DM continua fragmentada, descontínua e que o rastreamento do pé diabético, recomendado pelo Ministério da Saúde, é pouco realizado ou feito de maneira incompleta. Espera-se que esses resultados impulsionem práticas de enfermagem holísticas e políticas promocionais de saúde efetivas, visando reduzir o número de complicações decorrentes do DM.

Destaca-se como limitação deste estudo o fato de alguns pacientes com DM apresentarem exames laboratoriais desatualizados, o que inviabilizou a coleta de algumas variáveis clínicas que poderiam ser fatores de risco para o desenvolvimento do pé diabético.

\section{CONCLUSÃO}

Identificou-se que a situação conjugal com companheiro, o menor tempo de diagnóstico do DM e o menor número de complicações nos pés se mostraram fatores de proteção para o desenvolvimento do pé diabético. Em contrapartida, a obesidade, a hipertensão arterial, o tabagismo, o controle inadequado dos níveis de glicemia capilar, a não disposição para cuidar dos pés e a não realização do autoexame dos pés com frequência foram fatores de risco para o desenvolvimento do pé diabético. Nessa perspectiva, ressalta-se a importância de uma linha de cuidados efetiva, na Atenção Primária, voltada ao rastreamento do pé diabético e ao autocuidado, a fim de garantir ações assistenciais sistematizadas e preventivas.

No intuito de abrir caminhos para novas investigações, auxiliar no fortalecimento de políticas públicas voltadas aos pacientes com DM e instigar modificações na assistência, sugere-se: a realização do exame clínico dos pés como rotina na assistência às pessoas com DM, visando reduzir o número de ulceração; a aplicação da estratificação do risco de pé diabético, para facilitar o seguimento da assistência; o reforço de intervenções educativas voltadas para o autocuidado, objetivando diminuir as complicações nos pés; a disponibilização, aos enfermeiros da Atenção Primária, de materiais necessários para a realização do exame clínico completo dos pés no atendimento aos pacientes com DM.

\section{RESUMO}

Objetivo: Analisar os fatores associados ao risco de pé diabético em pessoas com diabetes mellitus atendidas na Atenção Primária. Método: Estudo observacional, analítico e transversal realizado em Teresina, Piauí, com pessoas diabéticas atendidas na Atenção Primária. A coleta de dados ocorreu mediante entrevista, exame clínico dos pés e análise do prontuário. Os dados foram analisados utilizando os testes estatísticos Mann-Whitney, Qui quadrado de Pearson e regressão logística múltipla. A força de associação entre as variáveis categóricas foi aferida pela Odds Ratio. Resultados: Participaram 322 pessoas. A situação conjugal com companheiro apresentou fator de proteção $(p=0,007)$. A hipertensão arterial $(p=0,045)$, obesidade $(p=0,011)$, tabagismo $(p=0,027)$, não ter sido submetido ao rastreamento $(\mathrm{p}=0,046)$, o controle inadequado da glicemia capilar $(\mathrm{p}<0,001)$, a não disposição para cuidar dos pés $(p=0,014)$ e a não realização do autoexame dos pés com frequência $(p=0,040)$ se mostraram fatores de risco para o desenvolvimento do pé diabético. Conclusão: Os aspectos sociodemográficos, clínicos e autocuidado interferem no risco de desenvolvimento do pé diabético, destacando a necessidade do rastreamento e de intervenções educativas eficientes para pessoas com diabetes mellitus na Atenção Primária.

\section{DESCRITORES}

Diabetes Mellitus; Pé Diabético; Programas de Rastreamento; Atenção Primária à Saúde; Enfermagem. 


\section{RESUMEN}

Objetivo: Analizar los factores asociados al riesgo de pie diabético en personas con diabetes mellitus en la Atención Primaria. Método: Se trata de un estudio observacional, analítico y transversal realizado en Teresina, Piauí, con personas diabéticas en la Atención Primaria. La recogida de datos se realizó mediante entrevistas, examen clínico de los pies y análisis de las historias clínicas. Los datos se analizaron por medio de las pruebas estadísticas de Mann-Whitney, Qui cuadrado de Pearson y regresión logística múltiple. La fuerza de la asociación entre las variables categóricas se midió con la Razón de Momios (Odds Ratio). Resultados: Participaron 322 personas. La situación marital con compañero se presentó como un factor de protección ( $\mathrm{p}=0,007)$. La hipertensión arterial $(\mathrm{p}=0,045)$, la obesidad $(\mathrm{p}=0,011)$, el tabaquismo $(\mathrm{p}=0,027)$, el no haber sido sometido a seguimientos $(\mathrm{p}=0,046)$, el control inadecuado de la glicemia capilar $(\mathrm{p}<0,001)$, la falta de disposición para cuidarse los pies $(\mathrm{p}=0,014)$ y la no realización del autoexamen de los pies a menudo ( $\mathrm{p}$ $=0,040)$ se revelaron como factores de riesgo para el desarrollo del pie diabético. Conclusión: Los aspectos sociodemográficos, clínicos y de autocuidado interfieren en el riesgo de desarrollar pie diabético, lo que pone de manifiesto la necesidad de rastreos e intervenciones educativas eficaces para las personas con diabetes mellitus en la Atención Primaria.

\section{DESCRIPTORES}

Diabetes Mellitus; Pie Diabético; Tamizaje Masivo; Atención Primaria de Salud; Enfermería.

\section{REFERÊNCIAS}

1. Brasil. Ministério da Saúde. Secretaria de Atenção à Saúde. Departamento de Atenção Básica. Manual do pé diabético: estratégias para o cuidado da pessoa com doença crônica [Internet]. Brasília, DF: Ministério da Saíde; 2016 [cited 2020 Mar 10]. Available from: http://189.28.128.100/dab/docs/portaldab/publicacoes/manual_do_pe_diabetico.pdf

2. Toscano CM, Sugita TH, Rosa MQ, Pedrosa HC, Rosa RD, Bahia LR. Annual direct medical costs of diabetic foot disease in Brazil: a cost of illness study. Int J Environ Res Public Health. 2018;15(1):1-13. https://doi.org/10.3390/ijerph15010089

3. Jiménez S, Rubio JA, Álvarez J, Ruiz-Grande F, Medina C. [Trends in the incidence of lower limb amputation after implementation of a Multidisciplinary Diabetic Foot Unit]. Endocrinol Diabetes Nutr. 2017;64(4):188-97. Spanish. https://doi.org/10.1016/j.endinu.2017.02.009

4. Marques AD, Silva LM, Moreira TM, Torres RA. Association between hospitalization due to diabetes mellitus and diabetic foot amputation. Enfermería Global. 2018;17(51):248-57. https://doi.org/10.6018/eglobal.17.3.286181

5. Silva HG, Lopes RM, Feitosa MC, Sousa KF, Oliveira RA. [Quality of life evaluation in type 2 diabetic patients and sensitive deficit prevalence in lower extremity]. Rev Bras Qual Vida. 2017;9(2):165-77. Spanish. https://doi.org/10.3895/rbqv.v9n2.6033

6. Netten JJ, Price PE, Lavery LA, Monteiro-Soares M, Rasmussen A, Jubiz Y, et al. Prevention of foot ulcers in the at-risk patient with diabetes: a systematic review. Diabetes Metab Res Rev. 2016;32(1 Suppl 1):84-98. https://doi.org/10.1002/dmrr.2701

7. Dutra LM, Novaes MR, Melo MC, Veloso DL, Faustino DL, Sousa LM. Assessment of ulceration risk in diabetic individuals. Rev Bras Enferm. 2018;71(2):733-9. https://doi.org/10.1590/0034-7167-2017-0337

8. Oliveira PS, Bezerra EP, Andrade LL, Gomes PL, Soares MJ, Costa MM. Practice nurse family health strategy in the prevention of diabetic foot. Rev Fund Care Online. 2016;8(3):4841-9. https://doi.org/10.9789/2175-5361.2016.v8i3.4841-4849

9. Menezes LC, Guedes MV, Moura NS, Moura DJ, Vieira LA, Barros AA. Knowledge of nurses in primary health care on the care for the diabetic foot. Estima. 2017;15(2):100-6. Portuguese. https://doi.org/10.5327/Z1806-3144201700020006

10. Mello RF, Pires ML, Kede J. Clinical evalu[ation form of lower members for diabetic foot prevention]. Rev Fund Care Online. 2017;9(3):899-913. Portuguese. https://doi.org/10.9789/2175-5361.rpcfo.v9.5468

11. Galdino YL, Moreira TM, Marques AD, Silva FA. Validation of a booklet on self-care with the diabetic foot. Rev Bras Enferm. 2019;72(3):817-24. https://doi.org/10.1590/0034-7167-2017-0900

12. Oliveira FB, Botelho KK, Bezerra AR, Azevedo DI, Santos-Couto-Paz CC, Fachin-Martins F. Cross-cultural adaptation to Brazilian Portuguese of the Michigan Neuropathy Screening Instrument: MNSI-Brazil. Arq Neuro-Psiquiat. 2016; 74(8):653-61. https://doi. org/10.1590/0004-282X20160094

13. Baldassaris ML, Martínez BB. [Cross-cultural adaptation of the diabetic foot examination instrument in 3 minutes]. Rev Bras Med Fam Comunidade. 2020;15(42):1-12. Portuguese. https://doi.org/10.5712/rbmfc15(42)2008

14. Katz MH. Multivariable analysis: a primer for readers of medical research. Ann Intern Med. 2003;138(8):644-50. https://doi. org/10.7326/0003-4819-138-8-200304150-00012

15. Garson GD. Structural equation modeling. Asheboro: Statistical Publishing Associates; 2010.

16. Teston EF, Senteio JS, Ribeiro BMSS, Maran E, Marcon SS. Risk factors for foot ulceration in individuals with type 2 Diabetes Mellitus. Cogitare Enferm. 2017; 22(4):e.51508. https://doi.org/10.5380/ce.v22i4.51508

17. Tracey ML, McHugh SM, Fitzgerald AP, Buckley CM, Canavan RJ, Kearney PM. Risk factors for macro- and microvascular complications among older adults with diagnosed type 2 diabetes: findings from The Irish Longitudinal Study on Ageing. J Diabetes Res. 2016;2016(5975903):5975903. https://doi.org/10.1155/2016/5975903

18. Alshareef SM, Aldayel AY, AlKhathlan MA, Alduaij KO, Alshareef FG, Al-Harthi ME, et al. Diabetic patients in Saudi Arabia: the evaluation of glycemic control measures based on emergency department utilization and the percentages of adherence to the recommended followups for microvascular complications. Saudi Med J. 2019;40(3):271-6. https://doi.org/10.15537/smj.2019.3.23968

19. Cubas MR, Santos OM, Retzlaff EM, Telma HL, Andrade IP, Moser AD, et al. [Diabetic foot: orientations and knowledge about prevention care]. Fisioter Mov. 2013;26(3):647-55. Portuguese. https://doi.org/10.1590/S0103-51502013000300019

20. Qureshi MS, Iqbal M, Zahoor S, Ali J, Javed MU. Ambulatory screening of diabetic neuropathy and predictors of its severity in outpatient settings. J Endocrinol Invest. 2017;40(4):425-30. https://doi.org/10.1007/s40618-016-0581-y 
21. Canto ED, Ceriello A, Rydén L, Ferrini M, Hansen TB, Schnell O, et al. Diabetes as a cardiovascular risk factor: an overview of global trends of macro and micro vascular complications. Eur J Prev Cardiol. 2019;1(1):1-8. https://doi.org/10.1177/2047487319878371

22. Ferreira LT, Saviolli IH, Valenti VE, Abreu LC. Diabetes mellitus: hyperglycemia and its chronic complications. ABCS Health Sciences. 2011;36(3):182-8.

23. Schroeder EB, Bayliss EA, Daugherty SL, Steiner JF. Gender differences in cardiovascular risk factors in incident diabetes. Womens Health Issues. 2014;24(1):e61-8. https://doi.org/10.1016/j.whi.2013.09.008

24. Rossaneis MA, Andrade SM, Gvozd R, Pissinati PS, Haddad MD. Factors associated with glycemic control in people with diabetes mellitus. Cien Saúde Coletiva. 2019;24(3):997-1005. https://doi.org/10.1590/1413-81232018243.02022017

25. Zhong A, Li G, Wang D, Sun Y, Zou X, Li B. The risks and external effects of diabetic foot ulcer on diabetic patients: a hospital-based survey in Wuhan area, China. Wound Repair Regen. 2017;25(5):858-63. https://doi.org/10.1111/wrr.12589

26. Rezende Neta DS, Silva AR, Silva GR. [Adhesion de las personas con diabetes mellitus autocuidado con sus pies]. Rev Bras Enferm. 2015;68(1):111-6. Portuguese. https://doi.org/10.1590/0034-7167.2015680115p

27. Lima RF, Fontbonne A, Carvalho EM, Montarroyos UR, Barreto MN, Cesse EA. Factors associated with glycemic control in people with diabetes at the Family Health Strategy in Pernambuco. Rev Esc Enferm USP. 2016;50(6):937-45. https://doi.org/10.1590/s0080623420160000700009

28. Couselo-Fernández I, Rumbo-Prieto JM. [Diabetic foot risk and self-care shortfalls among Type 2 Diabetes Mellitus patients]. Enferm Univ. 2018;15(1):17-29. Spanish. https://doi.org/10.22201/eneo.23958421e.2018.1.62902

29. Chin YF, Huang TT, Hsu BR, Weng LC, Wang CC. Factors associated with foot ulcer self-management behaviours among hospitalised patients with diabetes. J Clin Nurs. 2019;28(11-12):2253-64. https://doi.org/10.1111/jocn.14822

30. Sá PN, Moura JR, Melo Júnior EB, Almeida PC, Macêdo SF, Silva AR. Knowledge, attitudes and practices for the prevention of diabetic foot. Rev Gaúcha Enferm. 2014;35(3):36-42. https://doi.org/10.1590/1983-1447.2014.03.45187

Coordenação de Aperfeiçoamento de Pessoal de Nível Superior (Capes). Processo n. 88887.200647/2018-

00. Fundação de Amparo à Pesquisa do Estado do Piauí. Processo n. 88887.200647/2018-00. 